\title{
Clinical evaluation of the therapeutic effects of atelocollagen absorbable punctal plugs
}

This article was published in the following Dove Press journal:

Clinical Ophthalmology

13 January 2012

Number of times this article has been viewed

\author{
Kaori Hirai ${ }^{1,4}$ \\ Yoji Takano ${ }^{2}$ \\ Eiichi Uchio ${ }^{3}$ \\ Kazuaki Kadonosono 4 \\ 'Department of Ophthalmology, \\ International Goodwill Hospital, \\ ${ }^{2}$ Department of Ophthalmology, \\ Kitasato Institute Hospital, \\ ${ }^{3}$ Department of Ophthalmology, \\ Fukuoka, Fukuoka, Japan; ${ }^{4}$ Department \\ of Ophthalmology, Yokohama City \\ University Medical Center, \\ Yokohama, Japan
}

Purpose: To evaluate the therapeutic effects of atelocollagen absorbable punctal plugs.

Method: Seventy-four eyes in 37 patients with dry eye disease (DED) underwent punctal plug occlusion using atelocollagen plugs and were followed up for 24 weeks. Subjective symptoms were evaluated using the ocular surface disease index (OSDI) questionnaire. Ocular surface disease parameters, including the Schirmer test, phenol red thread test, tear break-up time (TBUT), and fluorescein and Rose Bengal staining, were recorded before and 1, 4, 8, 16, and 24 weeks after treatment.

Results: In comparison with the pretreatment data, the subjective symptoms and ocular surface disease parameters showed significant improvement after atelocollagen punctal occlusion. The average time of relapse was $17.4 \pm 5.1$ weeks. No severe complications occurred, and none of the plugs had to be removed because of local discomfort or epiphora.

Conclusion: Atelocollagen absorbable punctal plugs effectively improved ocular surface disorders in DED. These plugs are a safe and effective alternative in the treatment of DED.

Keywords: atelocollagen, absorbable punctual plug, dry eye

\section{Introduction}

A variety of pharmacologic and non-pharmacologic approaches exist for the management of dry eye disease (DED). Treatment is typically selected based on clinical signs and symptoms. Artificial tears are the mainstay of treatment and can be combined with hyaluronate sodium, autologous serum eye drops, and/or lacrimal outflow occlusion. In cases where local treatment does not ameliorate the condition, punctal plugs have been recognized as an effective, safe, and simple treatment. ${ }^{1-3}$ There are two main types of punctal plugs: nonabsorbable and absorbable. Nonabsorbable plugs have been used widely in the treatment of dry eye disease, ${ }^{2-4}$ however complications, such as spontaneous loss or epiphora (tear outflow), formation of granulomas, intracanalicular migration, and infections have been reported. ${ }^{2-5}$

In this study, we present the clinical results of a new absorbable punctual plug made of atelocollagen (Keeptear; Koken Bioscience Institutes, Tokyo, Japan). Atelocollagen is a collagen solution that is extracted from animal dermal tissue, from which antigenic telopeptides attached to both ends of the collagen molecule are eliminated by pepsin treatment. At temperatures of $4^{\circ} \mathrm{C}$ or under, atelocollagen dissolves in a neutral phosphate buffer solution. When heated to a temperature of $37^{\circ} \mathrm{C}$, the atelocollagen begins to solidify and turns into a white-colored gel that can be picked up using forceps. The plug therefore solidifies due to body temperature after injection through the punctum. ${ }^{3,6,7}$
Correspondence: Kaori Hirai I-28-I Nishigaoka Izumi-ku, Yokohama, Kanagawa, 245-0006, Japan

Tel $+8 \mid 458130221$

Fax +81458137419

Email hirai-kaori@shinzen.jp 
A PubMed search revealed that long-term follow-up data for atelocollagen absorbable punctal plugs has not yet been reported. Here, we report the follow-up data obtained during a 24-week evaluation of the therapeutic occlusion of lacrimal ducts using absorbable atelocollagen punctal plugs.

\section{Materials and methods}

The subjects were 74 eyes of 37 patients (34 women, 3 men; mean age \pm standard deviation (SD), $60.5 \pm 13.8$ years) diagnosed with dry eye disease (DED) at the Yokohama City University Medical Center from April 2009 to March 2010. The diagnosis of dry eye was based on the diagnostic criteria of the Dry Eye Research group in Japan. In brief, dry eye was diagnosed in patients who had (1) dry eye-related symptoms, (2) positive staining with fluorescein or Rose Bengal, and (3) Schirmer test results of less than $5 \mathrm{~mm}$, or tear break-up time (TBUT) of less than 5 seconds. None of the patients had a history of ocular surgery within 6 months prior to the study. None of the patients had previously undergone punctal occlusion. All patients gave informed consent before participation. This research protocol adhered to the tenets of the Declaration of Helsinki.

The patients had been previously treated with eye drops with unsatisfactory improvement in signs and symptoms. Prior to atelocollagen punctal occlusion, the patients had been using $0.1 \%$ sodium hyaluronate eye drops (Santen Pharmaceuticals, Osaka, Japan) and/or preservative-free artificial tears (Santen Pharmaceuticals). Two patients were using autologous serum tears and two patients were using $0.02 \%$ fluorometholone eye drops (Santen Pharmaceuticals). The patients were allowed to continue concomitant treatment with such eye drops during the study but were asked not to use any further eye drops without consulting the physician. Systemic drugs influencing tear production were prohibited during the study period. The patient backgrounds were Sjögren's syndrome (SS) (seven cases), post-laser in situ keratomileusis (LASIK) (two cases), and soft contact lens users (two cases). The treatment effects were evaluated using the Schirmer test (without local analgesia), the phenol red thread test, the tear break-up time (TBUT), and fluorescein and Rose Bengal staining. Fluorescein and Rose Bengal have traditionally been the dyes used for evaluation of the ocular surface. Fluorescein stains areas where the epithelium is absent, while Rose Bengal stains areas where the integrity of the mucin layer has been compromised. ${ }^{8}$ Fluorescein and Rose Bengal staining was graded from 0 to 3 at the nasal conjunctiva, temporal conjunctiva, and cornea, and the sum of the three grades was considered as the score for each eye.
Subjective symptoms were evaluated using the ocular surface disease index (OSDI) questionnaire. ${ }^{9}$ The OSDI is a validated, 12-item questionnaire used to assess the subjective symptoms of ocular surface disease.

Patients were examined before and 1, 4, 8, 16, and 24 weeks after treatment. All complications, defined as acute infection of the lacrimal drainage system, granulomatous formation, canalicular stenosis, or plug removal by the physician because of epiphora or local discomfort, were recorded.

Atelocollagen used in this study was extracted from bovine epidermis, sterilized, and packed in a sterile tube by Koken Bioscience Institutes (Tokyo, Japan). With patients laying down in a supine position, a 27-gauge blunt needle attached to a sterile tube containing $0.2 \%$ atelocollagen solution was inserted $2-3 \mathrm{~mm}$ into a punctum, and atelocollagen solution was injected into the canaliculi by pushing a plunger and slowly retracting the needle to fill the canaliculus with atelocollagen. After insertion of the atelocollagen punctal plug, patients were asked to keep their eyes closed for approximately 15 minutes. The eyes were warmed with a disposable eye-warming mask during this time to hasten solidification of the atelocollagen, preventing any leakage from the lacrimal ducts during blinking.

Statistical comparisons of the mean values between groups were performed using the Mann-Whitney $U$ test. The treatment outcome was analyzed using the Wilcoxon test. A probability value $<0.05$ was considered statistically significant.

\section{Results}

Seventy-four eyes in 37 patients underwent atelocollagen absorbable punctal plug treatment.

The frequency of eye drop use after treatment decreased in 28 patients and stayed the same in nine patients when compared with pretreatment frequency. No patients required the addition of further new eye drops after the punctal plug procedure.

Before treatment, mean tear secretion measured by the Schirmer test was $3.89 \pm 0.47 \mathrm{~mm}$. Schirmer test values significantly increased to $6.30 \pm 0.33 \mathrm{~mm}$ at 1 week, $5.86 \pm 0.34 \mathrm{~mm}$ at 4 weeks, and $5.97 \pm 0.47 \mathrm{~mm}$ at 8 weeks after treatment $(P<0.05)$ (Figure 1$)$.

Mean tear volume measured by the phenol red thread test was $13.3 \pm 0.85 \mathrm{~mm}$ before treatment. Mean tear volume significantly increased to $22.9 \pm 1.10 \mathrm{~mm}$ at 1 week, $20.4 \pm 1.13 \mathrm{~mm}$ at 4 weeks, and $19.5 \pm 1.05 \mathrm{~mm}$ at 8 weeks after treatment $(P<0.05)$ (Figure 1). 


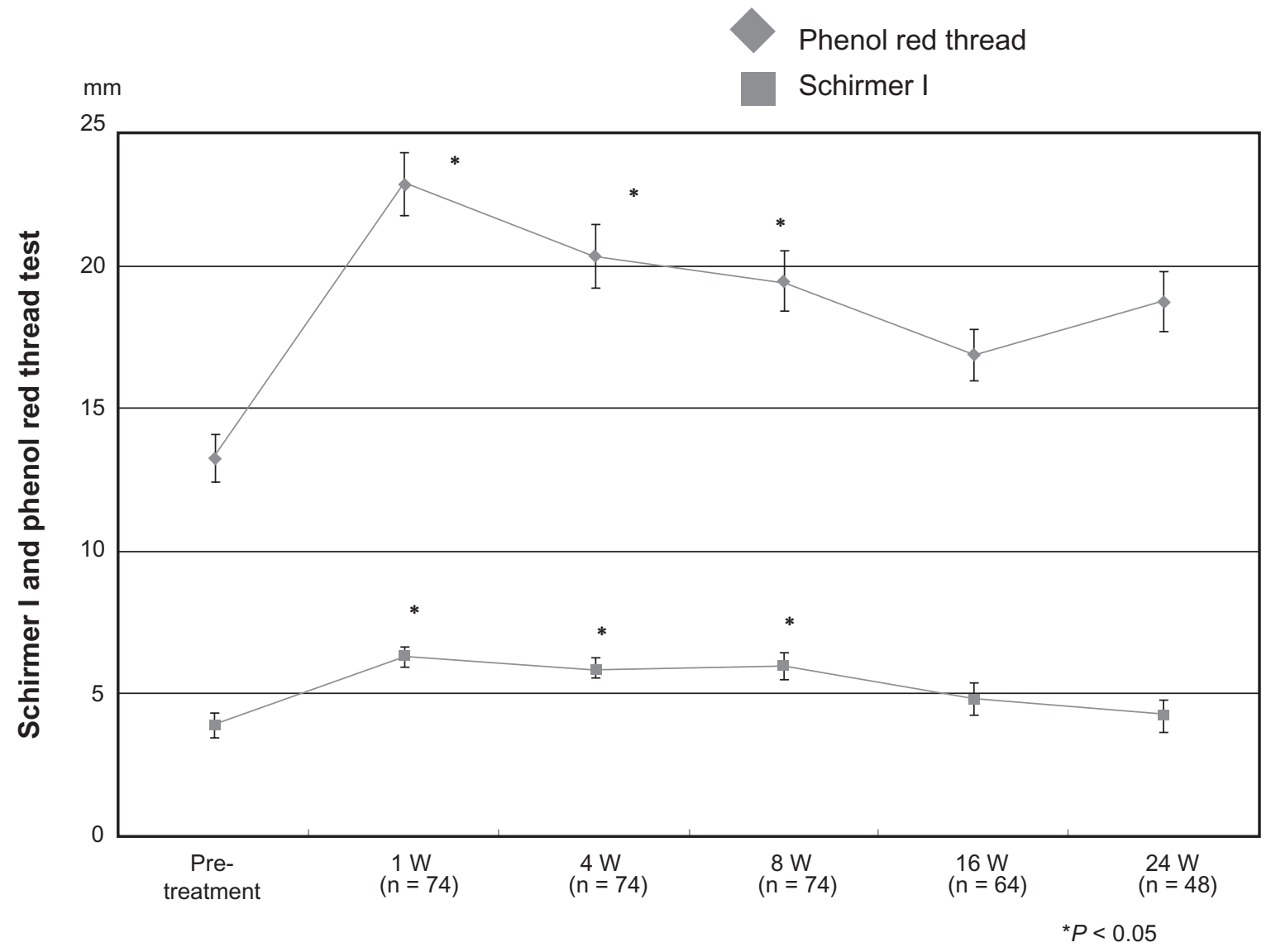

Figure I Schirmer and phenol red thread test results after treatment. Compared with the pretreatment levels, tear volumes measured using the Schirmer test (without analgesia) and the phenol-red thread test significantly increased at I week after treatment and slightly decreased thereafter.

Note: $* P<0.0001$ according to the Wilcoxon test.

Mean TBUT was $3.65 \pm 0.18$ seconds before treatment. Mean TBUT values increased after treatment, and were significantly higher at all time-points compared to pretreatment values $(P<0.05)$ (Figure 2).

Average fluorescein staining score was $2.62 \pm 0.20$ before treatment and decreased after treatment (Figure 3). All posttreatment values were significantly improved compared to pretreatment values $(P<0.05)$.

Average Rose Bengal staining score was $1.55 \pm 0.181$ before treatment and decreased after treatment (Figure 3). All post-treatment values were significantly improved compared to pretreatment values $(P<0.05)$.

The mean OSDI value decreased from $55.58 \pm 0.88$ before treatment and was significantly improved at all timepoints $(P<0.05)$. These findings suggest that the absorbable atelocollagen punctal plugs had a beneficial effect on the subjective symptoms (Figure 4).

In comparison with the pretreatment data, the subjective symptoms and ocular surface disease parameters (TBUT, fluorescein and Rose Bengal staining score) were significantly improved during the 24-week follow-up period. The improvement in tear volume measured with the Schirmer test and phenol red thread test was maintained for up to 8 weeks after treatment. These outcomes suggest that the absorbable atelocollagen punctal plugs had beneficial effects on both the objective and subjective symptoms.

None of the patients developed any severe adverse events related to the procedures, and none of the plugs had to be removed. Two patients complained of temporary local discomfort which was alleviated with the use of artificial tears.

Twenty-four eyes were reinjected with atelocollagen because of deteriorating symptoms during the follow-up period. The average time to relapse was $17.4 \pm 5.1$ weeks. The breakdown is; two eyes: 8 weeks, one eye: 12 weeks, seven eyes: 16 weeks, three eyes: 20 weeks, and four eyes: 24 weeks. No significant differences in the objective pretreatment parameters (tear volume, TBUT, fluorescein and Rose Bengal score) were observed between the retreatment group and the non-retreatment group. 


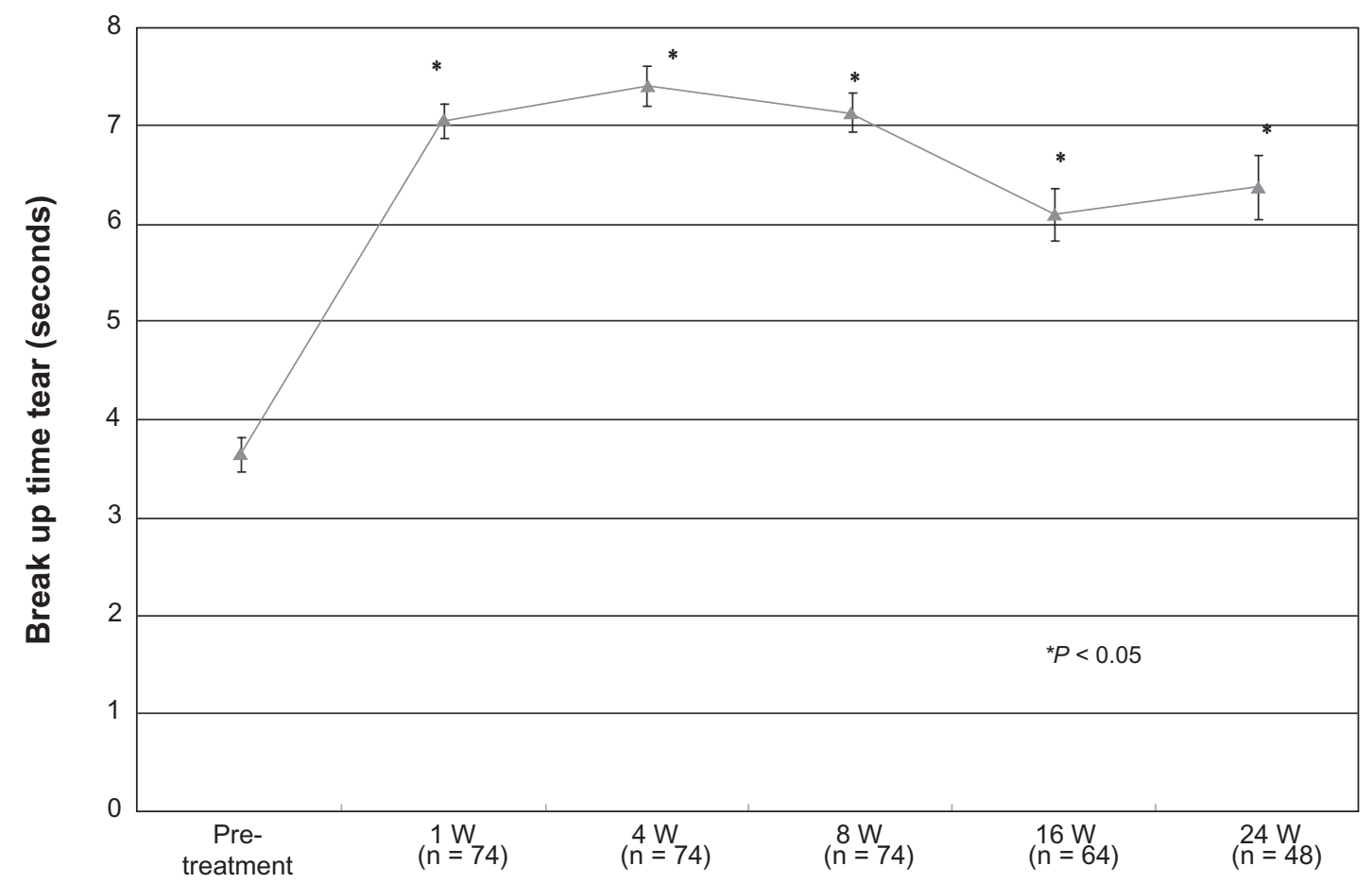

Figure 2 Tear break-up time (TBUT) after treatment. Compared with the pretreatment level, the mean TBUT time increased after treatment. Note: $* P<0.0001$ according to the Wilcoxon test.

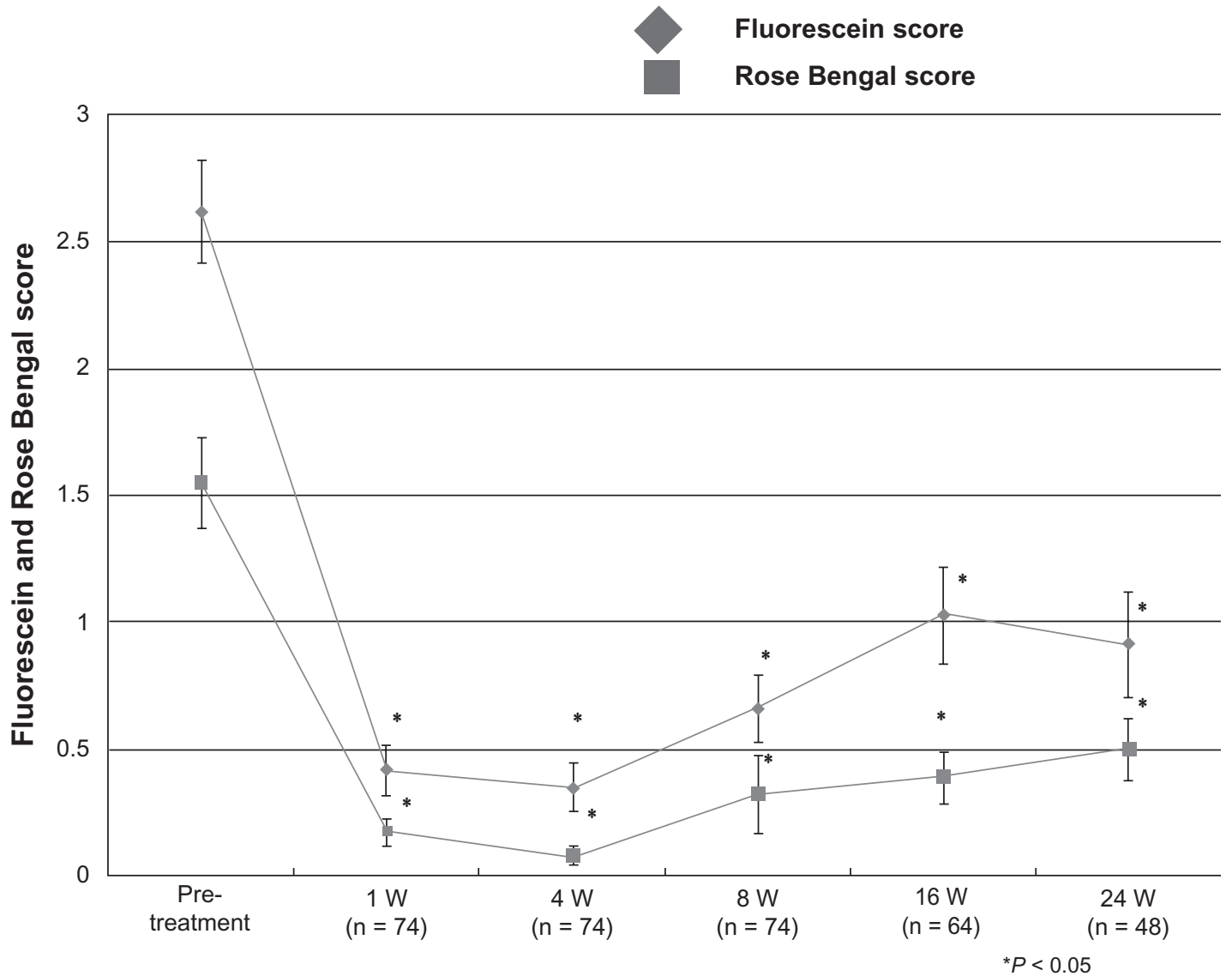

Figure 3 Fluorescein and Rose Bengal score after treatment. Compared with the pretreatment level, the fluorescein and Rose Bengal score decreased after treatment. Note: $* P<0.0001$ according to the Wilcoxon test. 


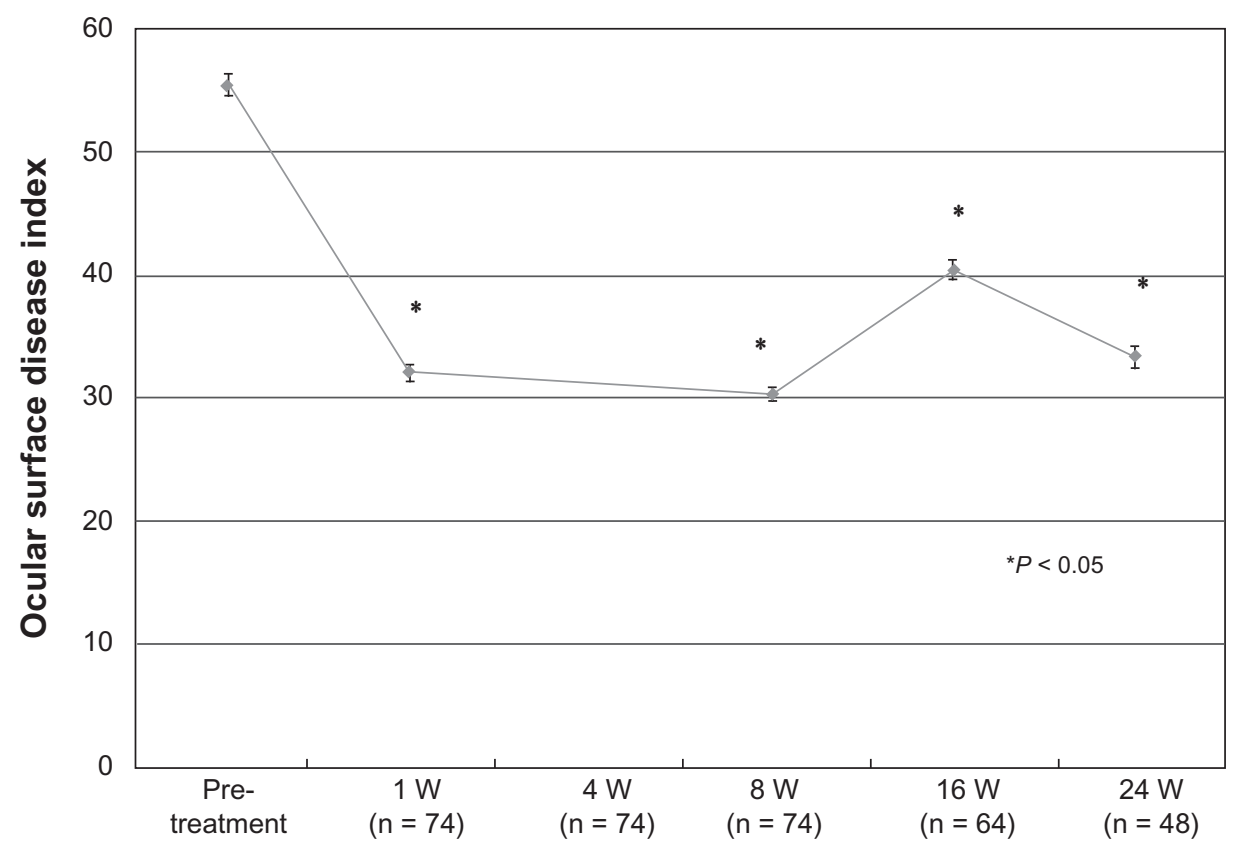

Figure 4 Ocular surface disease index (OSDI) after treatment. Compared with the pretreatment level, the OSDI decreased, and the subjective symptoms improved after treatment.

Note: $* P<0.0001$ according to the Wilcoxon test.

\section{Discussion}

We investigated the therapeutic effect and complications associated with absorbable atelocollagen punctal plugs. Vital staining with fluorescein and Rose Bengal, TBUT and OSDI were all significantly improved during the 24 -week period. The frequency of eye drop instillation was decreased in 28 of the 37 patients (76\%). No complications were observed suggesting that these plugs are safe and effective in the treatment of dry eye disease.

Improvement in Schirmer test and phenol red thread test values maintained significance up to 8 weeks but was not statistically significantly different after 16 weeks. The average time to relapse in reinjection cases was $17.4 \pm 5.1$ weeks. These findings suggest that the mean duration of efficacy of atelocollagen punctal plugs may be around 16 weeks.

Punctal plugs have offered a safe and often reversible treatment option for DED. The plugs are typically made of nonabsorbable (silicone, polyethylene, polymethylmethacrylate, and N-butyl cyanoacrylate) $)^{2,4,10}$ or absorbable (collagen, gelatin, catgut, or hydroxypropyl methyl-cellulose) $)^{3,11}$ materials. In recent years, the designs and models of punctal plugs have been improved, reducing the incidence of complications.

Complications of nonabsorbable punctal plugs, include spontaneous loss, acute conjunctivitis associated with biofilm formation, plug extrusion, epiphora, discomfort due to corneal or conjunctiva abrasion by the exposed portion of the plugs, and formation of granuloma. Intracanalicular migration may lead to canaliculitis and dacryocystitis. Canaliculitis and dacryocystitis caused by punctal plug migration may necessitate surgical procedures such as canaliculotomy or dacryocystorhinostomy (DCR) in severe cases. ${ }^{10,12-16}$

In contrast, as atelocollagen plugs solidify after injection into the canaliculi, prior size determination is unnecessary. This property also allows their use in cases with large puncta or cases with granulation that makes the insertion of conventional nonabsorbable plugs difficult. There is no risk of plug extrusion and the risk of granulomas and biofilm formation is minimal. The intracanalicular location of these plugs is also less likely to cause ocular surface irritation compared with conventional punctal plugs. ${ }^{3,4,10}$

The duration of action of absorbable punctal plugs is generally shorter than that of nonabsorbable punctal plugs. However the aforementioned advantages of atelocollagen plugs make it a safe and effective alternative to conventional nonabsorbable plugs. These plugs may in particular be indicated for cases with repeated loss of plugs, large puncta, and granuloma making conventional plug insertion difficult. These plugs may also be used initially to predict which patients are likely to tolerate nonabsorbable plugs or to treat a transient worsening of DED.

Atelocollagen is extracted from bovine dermis and an enzymatic process removes the antigenic portions from the collagen molecule. ${ }^{67}$ Atelocollagen is obtained from the 
dermis of Australian bred calves under the strictest conditions of safety. There have been no reported cases of bovine spongiform encephalopathy (BSE) in Australia. Moreover calf dermis is known to be a tissue free from infection by BSE prions. The source calves are strictly monitored from birth up to the point that the dermis is obtained and dermis is obtained from calves under 6 months which is well below the earliest onset of BSE at 30 months. Therefore the risk of prion infection due to the use of atelocollagen is unlikely.

In order to further assess the efficacy of this treatment modality more stringently, a controlled study with sham plug insertion as the control group is warranted.

In conclusion, atelocollagen absorbable punctal plugs effectively improved ocular surface disorders in DED. These plugs are a safe and effective alternative in the treatment of DED.

\section{Disclosures}

The authors report no conflicts of interest in this work. There was no financial support and the authors have no proprietary interest in the materials used in this study.

\section{References}

1. Cohen EJ. Punctal occlusion. Arch Ophthalmol. 1999;117(3): 389-390.

2. Lemp MA. Management of dry eye disease. Am J Manag Care. 2008; 14(Suppl 3):S88-S101.

3. Hamano T. Lacrimal duct occlusion for the treatment of dry eye. Semin Ophthalmol. 2005;20(2):71-74.

4. Altan-Y R, Genoglu EA, Akova YA, Dursun D, Cengiz F, Akman A. Silicone versus collagen plugs for treating dry eye: results of a prospective randomized trial including lacrimal scintigraphy. Am J Ophthamol. 2005;140(1):88-93.

5. Rumelt S, Remulla H, Runin PA. Silicone punctal plug migration resulting in dacryocystitis and canaliculitis. Cornea. 1997;16(3):377-379.

6. Ochiya T, Nagahara S, Sano A, Itoh H, Terada M. Biomaterials for gene delivery: atelocollagen-mediated controlled release of molecular medicines. Curr Gene Ther. 2001;1(1):31-52.

7. Miyata T, Taira T, Noishiki Y. Collagen engineering for biomaterial use. Clin Mater. 1992;9(3-4):139-148.

8. Born AJ. The Doyne Lecture. Reflections on the tears. Eye (Lond). 1997;11(Pt 5):538-602.

9. Schiffman RM, Christianson MD, Jacobsen G, et al. Reliability and validity of the Ocular Surface Disease Index. Arch Ophthalmol. 2000; 118(5):615-621.

10. [No authors listed]. Management and therapy of dry eye disease: report of the Management and Therapy Subcommittee of the International Dry Eye Workshop. Ocul Surf. 5(2):163-178.

11. Knapp TR, Kaplan EN, Daniels JR. Injectable collagen for soft tissue augmentation. Plast Reconstr Surg. 1977;60(3):398-405.

12. Horwath-Winter J, Thaci A, Gruber A, Boldin I. Long-term retention rates and complication of silicone punctal plugs in dry eye. $\mathrm{Am} J$ Ophthalmol. 2007;144(3):441-444.

13. Balaram M, Schaumberg DA, Dana MR. Efficacy and tolerability outcomes after punctal occlusion with silicone plugs in dry eye syndrome. Am J Ophthalmol. 2001;131(1):30-36.

14. Sakamoto A, Kitagawa K, Tatami A. Efficacy and retention rate of two types of silicone punctal plugs in patients with and without Sjögren syndrome. Cornea. 2004;23(3):249-254.

15. Taban M, Chen B, Perry JD. Update on punctal plugs. Compr Ophthalmol Update. 2006;7(5):205-212.

16. Tai MC, Cosar CB, Cohen EJ, Rapuano CJ, Laibson PR. The clinical efficiency of silicone punctal plug therapy. Cornea. 2002;21(2): 135-139.

\section{Dovepress}

\section{Publish your work in this journal}

Clinical Ophthalmology is an international, peer-reviewed journal covering all subspecialties within ophthalmology. Key topics include: Optometry; Visual science; Pharmacology and drug therapy in eye diseases; Basic Sciences; Primary and Secondary eye care; Patient Safety and Quality of Care Improvements. This journal is indexed on

PubMed Central and CAS, and is the official journal of The Society of Clinical Ophthalmology (SCO). The manuscript management system is completely online and includes a very quick and fair peer-review system, which is all easy to use. Visit http://www.dovepress.com/ testimonials.php to read real quotes from published authors. 